\title{
Civilisations
}

Revue internationale d'anthropologie et de sciences

humaines

66 | 2017

L'alcool rituel et les ethnographes

\section{Faire circuler la bière}

L'alcool comme force motrice et relationnelle dans une fête patronale bolivienne

\section{Laura Fléty}

\section{OpenEdition}

\section{Journals}

Édition électronique

URL : http://journals.openedition.org/civilisations/4565

DOI : 10.4000/civilisations.4565

ISSN : 2032-0442

\section{Éditeur}

Institut de sociologie de l'Université Libre de Bruxelles

\section{Édition imprimée}

Date de publication : 31 août 2017

Pagination : 177-194

ISBN : 978-2-9602017-1-0

ISSN : 0009-8140

Référence électronique

Laura Fléty, «Faire circuler la bière », Civilisations [En ligne], 66 | 2017, mis en ligne le 31 août 2020, consulté le 25 février 2021. URL : http://journals.openedition.org/civilisations/4565 ; DOI : https:// doi.org/10.4000/civilisations.4565 


\title{
Faire circuler la bière L'alcool comme force motrice et relationnelle dans une fête patronale bolivienne
}

\author{
Laura FLETY
}

Résumé : A partir d'une analyse de la circulation de la bière au sein d'un groupe de danseurs urbains d'origine aymara (Bolivie), cet article propose d'explorer la performativité rituelle et sociale de la consommation d'alcool. C'est à travers la mise en place de dispositifs spatiocorporels spécifiques que l'alcool est échangé, afin de produire un subtil jeu de rivalités et d'alliances qui fonde toute l'organisation sociale de ce collectif.

L'intention de cet article est de saisir au plus près des interactions et de l'expérience sensible, comment au sein de la fête l'alcool est non seulement l'objet central d'une dynamique de dons réciproques, mais aussi une substance de transformation corporelle intrinsèquement liée à la pratique dévotionnelle. Penser l'efficacité rituelle de l'alcool nécessite d'interroger l'articulation entre les pratiques sensori-motrices sur lesquelles reposent cette intense circulation de liquide et la manière dont les transactions d'alcool construisent les relations dans le groupe de danseurs.

Mots-clés : Corps, alcool, circulation, interactions, Andes, Bolivie.

\begin{abstract}
Based on an analysis of the circulation of beer in a group of urban dancers of Aymara origin (Bolivia), this article aims at exploring the ritual and social performativity of alcohol consumption. Alcohol is exchanged through the construction of specific spatial and corporal mechanisms to produce a subtle game of rivalry and alliances that underpins the whole social organization of this collective.

From the study of interactions and sensitive experiences, this article will investigate how alcohol is not only the central object of a dynamic of reciprocal gifts within the festivity, but also a substance of transformation of the body intrinsically linked to devotional practice. In order to comprehend the ritual efficiency of alcohol, one has to question the articulation between corporal practices - on which this intense circulation of liquid relies - and the way alcohol transactions help building relationships within the group of dancers.
\end{abstract}

Keywords: Body, alcohol, circulation, interactions, Andes, Bolivia. 


\section{Introduction}

Au sein des fêtes rituelles des communautés indigènes boliviennes, danse, musique et abondance d'alcool apparaissent comme les éléments fondateurs d'une organisation sensorielle complexe où prime l'expérience de la saturation des sens ${ }^{1}$. Cet « enivrement » des participants à travers les sons, les mouvements et la forte consommation d'alcool est conçu comme indispensable à la circulation de l'énergie de l'univers (Arnold 1992 : 117-119) et à la communication avec le monde invisible ${ }^{2}$ (Saignes 1993 ; Martínez 2002 ; Geffroy 2013).

Ce phénomène de saturation multi-sensorielle s'observe également dans les grandes fêtes urbaines de la Bolivie contemporaine qui célèbrent diverses vierges et saints patrons $^{3}$. La célébration annuelle de Jesús del Gran Poder ${ }^{4}$, le Christ protecteur de la ville de La Paz, rassemble plusieurs milliers de danseurs et de musiciens dans un somptueux cortège défilant jusqu'à épuisement. Dans leurs costumes aux couleurs éclatantes, les groupes de danseurs avancent sous forme de bataillons dans les rues de la ville, dans une ivresse partagée entre ceux qui font la performance et ceux qui la regardent. De l'église, point de départ des cortèges, jusqu'à la fin du parcours, s'opère une transformation progressive des corps des danseurs, des musiciens et des spectateurs. Le défilé s'achève dans une forme d' " excès " (Tassi 2010) ${ }^{5}$, matérialisé à la fois par l'intensité sonore et gestuelle et par la circulation continue de liquides ${ }^{6}$ : bière, urine, transpiration. L'espace urbain du quotidien devient l'espace du rituel où performance chorégraphique et alcool constituent les offrandes au Christ dont les danseurs espèrent en retour recevoir les faveurs (chance, santé, travail et richesses).

A partir d'une description ethnographique ${ }^{7}$ de la circulation et de la consommation

1 H. Stobart (2002 : 114) suggère qu'au sein des rituels andins, les couleurs brillantes et les sons éclatants sont intimement associés à l'expression des énergies internes des corps, elles-mêmes dépendantes de l'intensité de la saturation multi-sensorielle atteinte pendant la fête. Sur ce sujet, voir également les travaux de C. Classen (1990) et R. Martínez (2009).

2 Le monde invisible est peuplé d'entités surnaturelles tels saints et vierges catholiques, entités démoniaques et divinités du panthéon andin. Parmi les plus importantes, la Pachamama (Terre-mère) est capable d'être cruelle et bienveillante. Le Tío - Dieu de la montagne et du minerai - est associé au Diable catholique, il incarne une figure dangereuse et source de richesses (Absi 2002).

3 Les principales grandes fêtes urbaines sont la Fiesta del Gran Poder (La Paz), le Carnaval de Oruro (Oruro), la Fiesta de la Vírgen de Urkupiña (Cochabamba), El Chutillo (Potosi) et la Fiesta de la Vírgen de Guadalupe (Sucre).

4 Celle-ci se déroule chaque année le samedi précédant la fête de la Sainte-Trinité.

5 Nico Tassi montre comment, à travers leurs défilés, les danseurs exposent une matérialité ostentatoire (2010 : 200-201). Tous ces éléments accumulés permettent aux danseurs d' « attirer» sur eux l'abondance qu'ils exhibent (ibid. : 196-197) et l' « excès » devient le principe constitutif d'une forme de « théologie matérielle » (ibid. : 206).

6 Sur l'importance de l'humidité en contexte festif andin comme état propice à la vie et au renouvellement (le sec renvoyant à la mort), voir Geffroy $(2012,2013)$.

7 Ces données sont issues d'une enquête de terrain réalisée entre 2008 et 2013, dans les quartiers nordouest de la ville de La Paz (Bolivie), au sein d'un groupe de danseurs appelé « Fraternidad Morenada Rosas de Viacha » (Fraternité Morenada Roses de Viacha) qui défile tous les ans pour la célébration du Christ Gran Poder. 
de bière au sein d'un groupe de danseurs urbains d'origine aymara ${ }^{8}$, cet article propose d'explorer la performativité rituelle, corporelle et sociale de la consommation d'alcool. Pour exprimer leur dévotion, ces danseurs exécutent la danse morenada, l'une des principales danses de la fête qui met en scène une troupe de morenos (littéralement " ceux qui ont la peau foncée »), personnages portant d'imposants costumes et de somptueux masques aux visages noirs et argentés. Ces derniers sont précédés d'une formation d'élégantes danseuses portant la pollera (jupe bouffante) et un chapeau melon porté en équilibre sur la tête, tenue vestimentaire typique des femmes métisses de La Paz, appelées cholas 9 .

Les danseurs de morenada sont majoritairement issus de la migration indigène ${ }^{10}$ de première et deuxième générations et se sont intégrés à la société urbaine en développant une économie informelle très dynamique autour du commerce et de l'artisanat (Tassi 2010). Leur participation massive au sein du défilé, mais également de manière indirecte en tant qu'artisans des costumes et masques, constitue sans doute l'un des traits sociologiques caractéristiques de cette fête. Installés depuis plusieurs dizaines d'années dans les anciens faubourgs indigènes du nord-ouest de La Paz, ils appartiennent aux couches supérieures de la population migrante ${ }^{11}$.

La fête du Christ protecteur de La Paz, qui s'ancra dans ces quartiers à partir des années 1920, fut considérée par l'élite de la ville comme une indécente beuverie collective et un symbole de sauvagerie et d'idolâtrie indienne (Guss 2006 : 320). Actuellement, malgré les injonctions et multiples sanctions encore imposées aux participants par l'Eglise catholique et le comité organisateur de la célébration, ces pratiques d'alcoolisation constituent encore un phénomène central de cet évènement. Avant et après la danse, les participants consomment plusieurs dizaines de litres de bière suivant les règles d'un protocole précis dans lequel chacun réinvite toujours la personne qui lui a déjà offert à boire $^{12}$.

Pour les danseurs, l'association entre dépense corporelle et consommation d'alcool constitue le moteur de la dévotion et permet de vivre « en actes » un principe vital

8 Les Aymaras sont l'un des principaux groupes linguistiques de Bolivie avec les Quechuas et les Guaranís. La population indigène aymara vit principalement autour du lac Titicaca ; La Paz et El Alto sont les principales villes qui accueillent les migrants de ces zones rurales et des exploitations minières de la région.

9 Les cholas, que l'on peut désigner schématiquement par « Indiennes en ascension sociale », se situent socialement entre les paysannes et les señoras (« dames de la ville ») (Marchand $2010: 222$ ).

10 Ils proviennent des villages et des communautés indigènes des provinces d'Ingavi, Omasuyos ou Pacajes qui appartiennent au département de La Paz et qui se situent aux alentours du lac Titicaca, entre la frontière de la Bolivie et du Pérou. Les plus âgés d'entre eux parlent encore les langues indigènes (généralement l'aymara mais aussi parfois le quechua).

11 Depuis une trentaine d'années, cette population migrante d'origine aymara a par ailleurs acquis une forte visibilité ainsi qu'un poids institutionnel et politique croissant, et ce, dans les universités, l'administration ou les médias, phénomène qui s'est accentué notamment depuis l'élection en 2005 du président d'origine aymara, Evo Morales.

12 Si dans d'autres contextes festifs (mariage, baptême, anniversaire) on retrouve ces formes d'invitations alternées et répétitives, celles-ci diffèrent de la consommation d'alcool plus quotidienne de ces danseurs, dans les bars ou chicherias (lieux où l'on prépare et consomme de la bière de maïs appelée chicha) où chacun paie ce qu'il consomme. Par ailleurs, la consommation d'alcool collective et ritualisée - telle que décrite dans cet article - est propre à l'univers socioculturel des aymaras urbains et contraste fortement avec les modes de consommation individualisés des classes urbaines moyennes/hautes boliviennes. 
de circulation (Fléty 2015). Intiment liées, la pratique dansée et celle de l'ivresse, impliquent un type d'expérience sensori-motrice qui relève d'un même registre pour les participants, celui de la fuerza (force), de la résistance à la fatigue, considérée comme nécessaire à l'exécution du rituel et à l'expression de la foi religieuse. Néanmoins, la construction d'une expérience socialement codifiée de l'alcoolisation dépasse l'acte dansé. D'une part, j'analyserai la manière dont on manipule et distribue l'alcool sous forme de dons réciproques : obligation de donner, de recevoir et de rendre (Mauss 1925) à travers un subtil jeu de rivalités et d'alliances sociales ${ }^{13}$. Pour être effectives ces transactions d'alcool doivent être réalisées dans des dispositions corporelles spécifiques, savoir s'《 agiter » pour offrir à boire à ses partenaires ou, au contraire, savoir être « immobile» pour recevoir les dons d'alcool. Dans ce texte, j'appréhenderai donc le corps « en action avec les objets » (Julien, Rosselin et Warnier $2006: 45$ ).

D'autre part, à ces obligations s'ajoute celle de consommer « ensemble » l'alcool, en grande quantité, ce qui modifie la corporéité ${ }^{14}$ des danseurs. Ainsi, contrairement à l'idée que l'importance du don est moins le « bien qui circule que le lien qui résulte de la transaction », idée avancée par Alain Caillé et Jacques Gotbout (cité par Albert 2011 : 15), la nature du bien qui circule dans notre cas, revêt une importance fondamentale. Au plus près des interactions et de l'expérience corporelle (Csordas 1994), ce texte montre comment dans un même espace-temps de la fête, l'alcool est à la fois l'objet central d'une dynamique de dons réciproques et une substance de transformation physique, intrinsèquement liée à la pratique dévotionnelle.

Penser l'efficacité rituelle de l'alcool nécessite une analyse tant de l'effet qu'il produit que des enjeux sociaux dont il est partie prenante. L'intérêt de cette réflexion réside dans le choix de combiner une approche qui s'intéresse tant à la manière dont les transactions d'alcool construisent les relations internes du groupe à différents niveaux, qu'à l'expérience sensori-motrice intrinsèquement liée à cette circulation : symétrie spatiale, qualité du mouvement, sensations kinesthésiques.

\section{Où l'alcool partagé acte une relation}

Le groupe de danseurs de morenada appelé fraternidad ${ }^{15}$ organise des activités religieuses et chorégraphiques internes (répétitions, veillées, messes) lors desquelles le même impératif s'impose : l'alcool doit être offert, consommé et rendu de manière ostentatoire, matérialisant l'idée d'abondance et de réciprocité. Le liquide n'est pas uniquement ingéré en grande quantité mais ses récipients sont utilisés dans une mise en scène qui focalise sur eux toute l'attention. Les caisses de bières sont placées au milieu de la rue, empilées en pyramide ou sous forme d'imposants murs. Leur consommation suit un scénario précis que tous les membres connaissent et qui met en relief les valeurs cardinales de ces rassemblements : la circulation et la dépense.

13 Comme le souligne J-P. Albert, l'enjeu du don est de l'ordre du prestige : « on donne pour se grandir, au moins pour tenir son rang » (2011:15).

14 D’inspiration phénoménologique, la « corporéité » élargit la notion de corps à l'ensemble de ce qui le constitue et l'anime, les émotions, les affects, l'expressivité (Bernard 1995).

15 Les fraternidades sont des structures associatives que l'on retrouve couramment dans les fêtes patronales des grandes villes andines et dont l'activité centrale est celle de danser en l'honneur d'un saint ou d'une vierge. Elles seraient inspirées des confréries religieuses implantées dans le Nouveau Monde lors de la colonisation (Celestino et Meyers 1981). 
Par ailleurs, au sein de la fraternidad toute consommation d'alcool est précédée d'une ch'alla, geste de libation rituelle qui consiste à verser une petite quantité d'alcool sur le sol avant de l'ingérer. C'est là une offrande à Jesús del Gran Poder, à la Pachamama (Terre-mère), aux saints, aux défunts ou autres divinités.

Or, si comme le suggère Randall (1993), les Andins sont impliqués dans une chaîne ininterrompue de dons, contre-dons et d'obligations envers les dieux, cette chaîne d'échanges est également présente au cœur des rapports sociaux des danseurs urbains. Pour chaque danseur, les transferts de bouteilles de bière engagent deux types de relations. D'une part, celles qui se jouent avec la «famille élargie », c'est-à-dire avec le cercle proche constitué des membres de la famille, des amis et de la parenté rituelle (relations de « comparrainage » (compadrinazgo) qui comprend l'ensemble des relations de parrainage et compérage $\left.{ }^{16}\right)$. D'autre part, les transferts d'alcool instituent la relation avec les autorités rituelles de la fraternidad, appelées pasantes. Il s'agit d'un ou plusieurs couples (mari et femme) qui prennent annuellement le « cargo » : la charge économique et rituelle ${ }^{17}$, c'est-à-dire l'organisation et les dépenses de la fête, accédant ainsi à un statut de grand prestige. Idéalement, l'investissement des pasantes dans la fraternidad doit être total, affectant vie professionnelle et familiale; il constitue simultanément un devoir envers le groupe et un acte de dévotion suprême envers Jesús del Gran Poder.

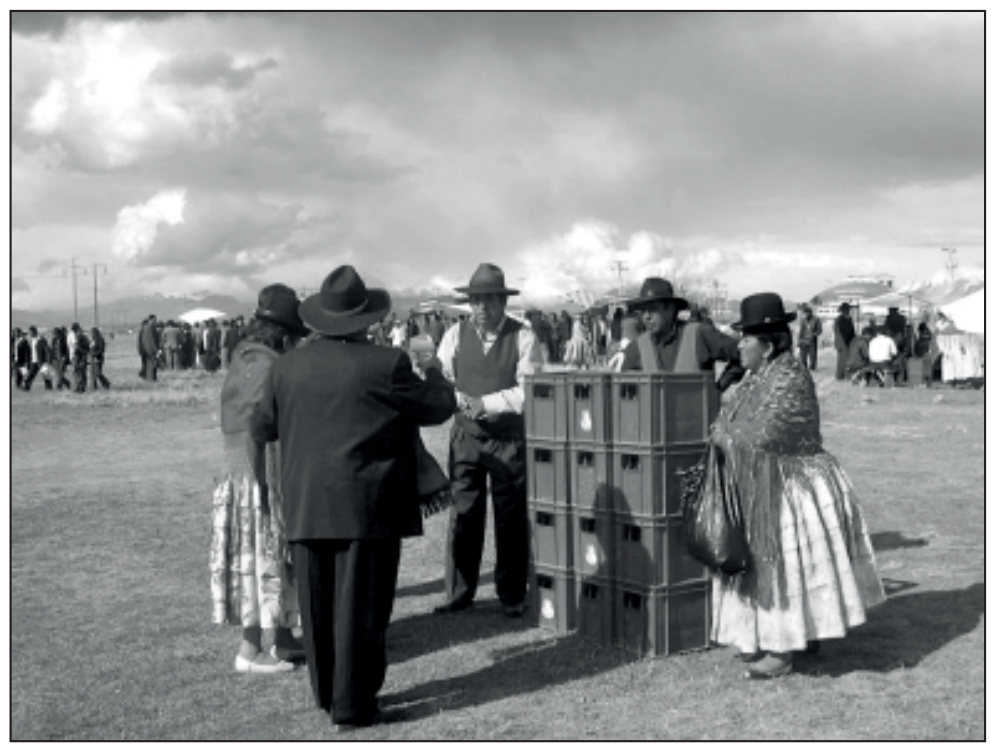

Figure 1 : Caisses de bières offertes aux pasantes lors d'une fête - C Laura Fléty

16 Premièrement, les danseurs peuvent être liés par une relation de compérage de type religieux. C'est une relation horizontale qui lie les parents aux parrains de leur enfant et qui devient effective lors du baptême de ce dernier. Deuxième cas de figure, les danseurs peuvent être liés par une relation de parrainage. C'est une relation verticale entre un couple et leurs parrains de mariage (padrinos de boda). Enfin, les danseurs peuvent être liés par une relation de compérage qui ne passe pas par un rite catholique mais qui témoigne d'une grande amitié ou affinité. Quelle que soit la nature du lien de parenté rituelle, il implique toujours des obligations de réciprocité et de respect équivalant à un lien de parenté matrimonial ou biologique.

17 On retrouve le modèle du système de charge dans toute l'Amérique hispanique, tant en milieu urbain que rural, il est l'un des fondements de l'organisation communautaire dite « traditionnelle » des populations andines. 


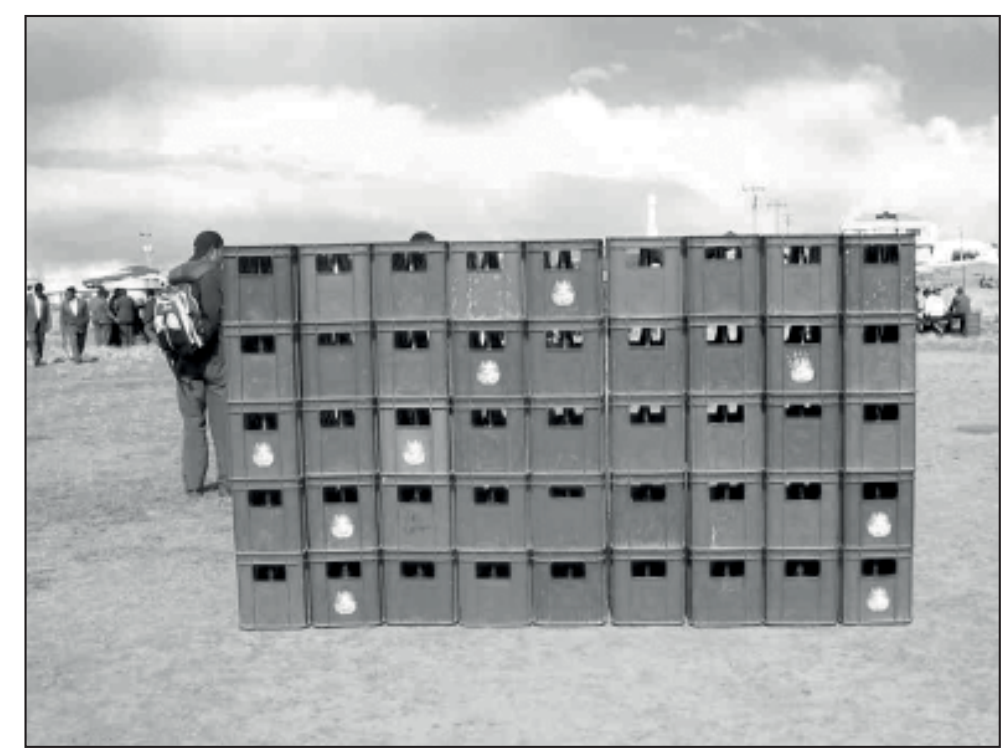

Figure 2 : Mur de caisses de bières offertes aux danseurs par les pasantes - (c) Laura Fléty

\section{Boire entre soi}

C'est généralement en famille que les danseurs, appelés fraternos (littéralement « frères »), participent aux activités chorégraphiques de leur fraternidad. Dans la rue, en attendant de défiler, ou lors des fêtes internes qui se déroulent dans des salons de danse privés, les danseurs d'un même réseau familial restent soudés en formant de petits cercles fermés autour de caisses de bières posées sur le sol qu'ils se partagent. Dès que la fanfare s'arrête de jouer, ils profitent du silence pour crier à l'unisson le nom qu'ils se sont auto-attribué : «Les Loups », « Les Infidèles », « Les Colombes en or », « Les Intrépides » ou encore « les Cœurs sauvages ». Ils accompagnent ces injonctions verbales de longs sifflements et de courts cris aigus d'un grand volume sonore. S'ensuit l'ouverture bruyante et emphatique d'une série de bouteilles de bière dont le jet explose et arrose abondamment le sol, marquant ainsi leur présence au sein de la fraternidad et attirant le regard de tous les autres danseurs présents. Quelques secondes plus tard, à quelques mètres, un autre petit groupe exécute exactement la même action, et ainsi de suite dans une dynamique de surenchère visuelle et sonore. L'alcool se sert avec toujours plus d'effusion et les bouteilles se vident à grande vitesse. Chaque famille (au sens élargi) affirme ainsi publiquement la force des liens qui les unissent et la joie d'être réuni pour danser.

Une attention fine aux manières de boire invite à penser que cette consommation n'a pas comme unique objectif l'expérimentation des propriétés enivrantes de l'alcool mais la mise en place d'un protocole selon lequel l'invitation à boire atteste, crée et renforce des liens sociaux primordiaux. Dans un même cercle, c'est d'abord une seule personne qui achète plusieurs bouteilles ou caisses et sert les uns après les autres tous les membres. Selon les danseurs, cet acte témoigne du cariño (marques d'affection) et du respect que l'on a pour un autre proche. Par exemple, un couple offrira obligatoirement à boire aux parrains de leurs enfants ou parrains de mariage et il est impensable que des danseurs liés par une relation de compérage ne s'offrent mutuellement de grande quantité d'alcool. 


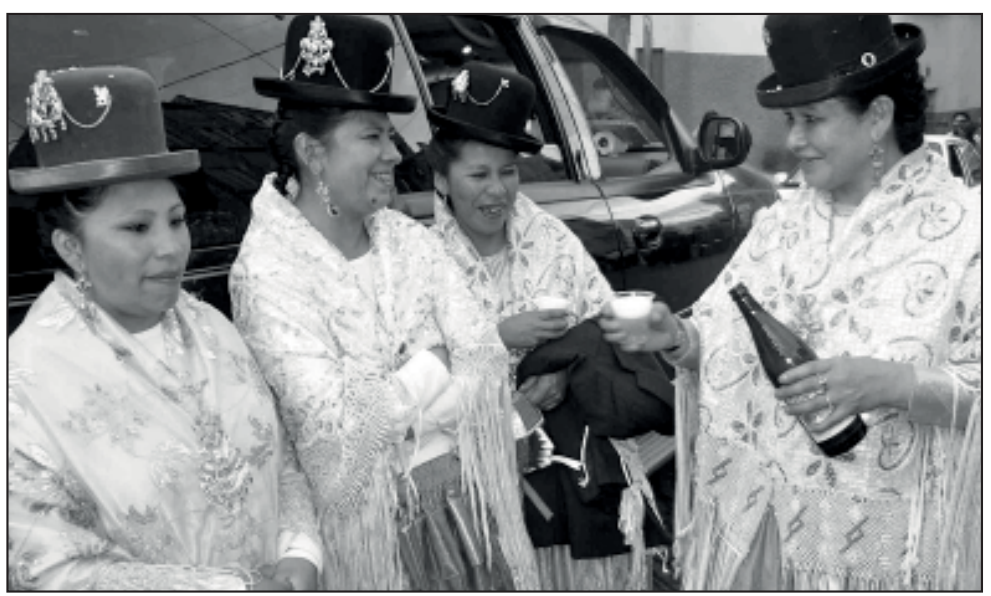

Figure 3 : Doña Maria offre à boire à ses comadres (commères) - C Laura Fléty

Au sein du groupe familial, chacun à son tour prend en charge les dépenses de l'alcool - cette activité est centrale dans toutes les interactions. Il n'existe aucun moment où les verres et bouteilles ne s'échangent pas. Ce qui caractérise cette intense circulation est principalement la générosité qu'elle met en scène. Pour être le premier à inviter une caisse de bières, on court expressément chercher un petit vendeur ambulant, on sort toujours promptement son portefeuille, on ne recompte jamais son argent... Prime ainsi l'idée qu'il faut toujours acheter et donner à boire avec largesse. Il est impératif de donner « sans compter », « sans calculer », attitude qui n'est par exemple pas présente dans d'autres activités comme les achats des costumes ou les échanges de services entre danseurs.

Cette posture renvoie de manière plus générale à l'idée que la dépense est nécessaire à la fête, que celle-ci soit physique (la danse) ou économique (achat des bières). L'exhibition collective de l'abondance révèle une façon tout à fait singulière de structurer le rituel par la mise en place d'une circulation des flux : l'argent est dépensé tour à tour et par chacun, les bouteilles pleines sont vidées puis remplacées, les corps transpirent pour danser, s'emplissent de liquides et se vidangent. Ce principe de circulation s'établit non seulement dans un entre-soi familial au sein duquel les alliances sont continuellement réaffirmées mais également à une autre échelle, celle de la relation avec les pasantes, ceux qui assument la charge rituelle de la fête.

\section{La relation aux pasantes : Ayni et circuit des caisses de bières}

Pour faire face aux lourdes dépenses liées à l'organisation de la fête, les pasantes mobilisent l'ensemble de leurs réseaux. A travers ce que les danseurs appellent le système des aynis, leur parentèle et leurs amis ont l'obligation de leur apporter un soutien. Dans la littérature anthropologique andiniste, le terme d'ayni réfère à un type de travail communautaire qui constitue la base primordiale des liens sociaux dans les processus de production (Wachtel 1990 : 563-568). C'est un modèle de coopération symétrique dans lequel s'échangent des prestations de même nature (services, travaux, activités agricoles) et de même quantité.

En milieu urbain et dans les fraternidades, on dit par exemple d'un pasante prestigieux qu'il a « beaucoup d'aynis », évoquant par-là l'aide apportée par ses proches. Cette aide 
se traduit par la participation dansée de chacun d'entre eux, à travers les nombreux cadeaux offerts ayant plus ou moins de valeur (argent liquide, parures, bijoux, bibelots souvenirs, etc.) et, principalement, par les importants dons de caisses de bières. Si par la suite, ceux qui ont apporté leur soutien deviennent à leur tour pasantes, ces contributions leur seront rendues de manière égale ou supérieure par ceux qu'ils auront précédemment aidés. Ce système entretient un idéal d'équivalence et de réciprocité collective au sein d'un groupe de parenté élargi et consolide les liens entre les danseurs, en même temps qu'il instaure une forme de dette. En effet, même si l'ayni est une forme de réciprocité symétrique, celle-ci instaure un déséquilibre qui ne sera compensé que dans le futur : les pasantes ne pourront rétablir l'équilibre avec ceux qui les ont aidés que dans un temps plus ou moins long.

Il convient déjà ici de distinguer une différence majeure entre les transferts d'alcool opérés entre membres d'un petit groupe familial, tels décris précédemment, avec ceux opérés entre pasantes et leurs cercles proches. Dans le premier cas, on offre à boire pour témoigner son affection, les invitations et contre-invitations d'alcool marquent l'intimité, le plaisir, et une fois la fête et la danse achevées, personne n'est en dette car la rétribution est immédiate. A l'inverse, les transferts de caisses de bières opérés entre danseurs et pasantes imposent un autre type de règles, les affinités sociales et la complicité n'étant pas absentes pour autant. Avec les dons d'alcool, les pasantes reçoivent le prestige et l'estime de la communauté mais devront rendre ensuite un service équivalent.

Si le contre-don n'est pas honoré, les sanctions seront informelles (perte de prestige, humiliation, réprobation), mais n'impliquent aucune obligation juridique, contrairement au cas de l' " échange » où celle-ci prévaut (Testart 2001). Néanmoins, les contre-dons sont dans l'ensemble toujours respectés car ils permettent de garder actif un réseau d'alliances et des relations pérennes. L'alcool est ici un don réciproque qui, comme le résume Gregory (1982), est un bien inaliénable qui instaure des relations entre des partenaires interdépendants ${ }^{18}$.

En outre, plus qu'une valeur purement morale, la réciprocité se concrétise à travers une série d'actions corporelles et de dispositifs spatiaux spécifiques dans lesquels l'alcool est au centre. Par exemple, les dons de caisses de bières que réalisent les danseurs pour les pasantes se font selon un déplacement spatial précis qui met en action la logique de la réciprocité. Les caisses de bières sont placées au milieu de la rue, empilées en pyramide, et ce sont les déplacements autour de cette construction qui activent et matérialisent le principe de l'ayni.

18 C. Gregory donne cette définition du don pour l'opposer au concept de « marchandises » qui instaurerait des relations entre partenaires indépendants. Cependant, dans la littérature sur le don, cette dichotomie classique entre économie de don et économie de marchandise a largement été remise en question par les travaux réunis par C. Humphrey et Hugh-Jones (1992) et par de nombreuses ethnographies andines. Sur cette question, voir le travail sur la Yapa d'O Angé (2011). 


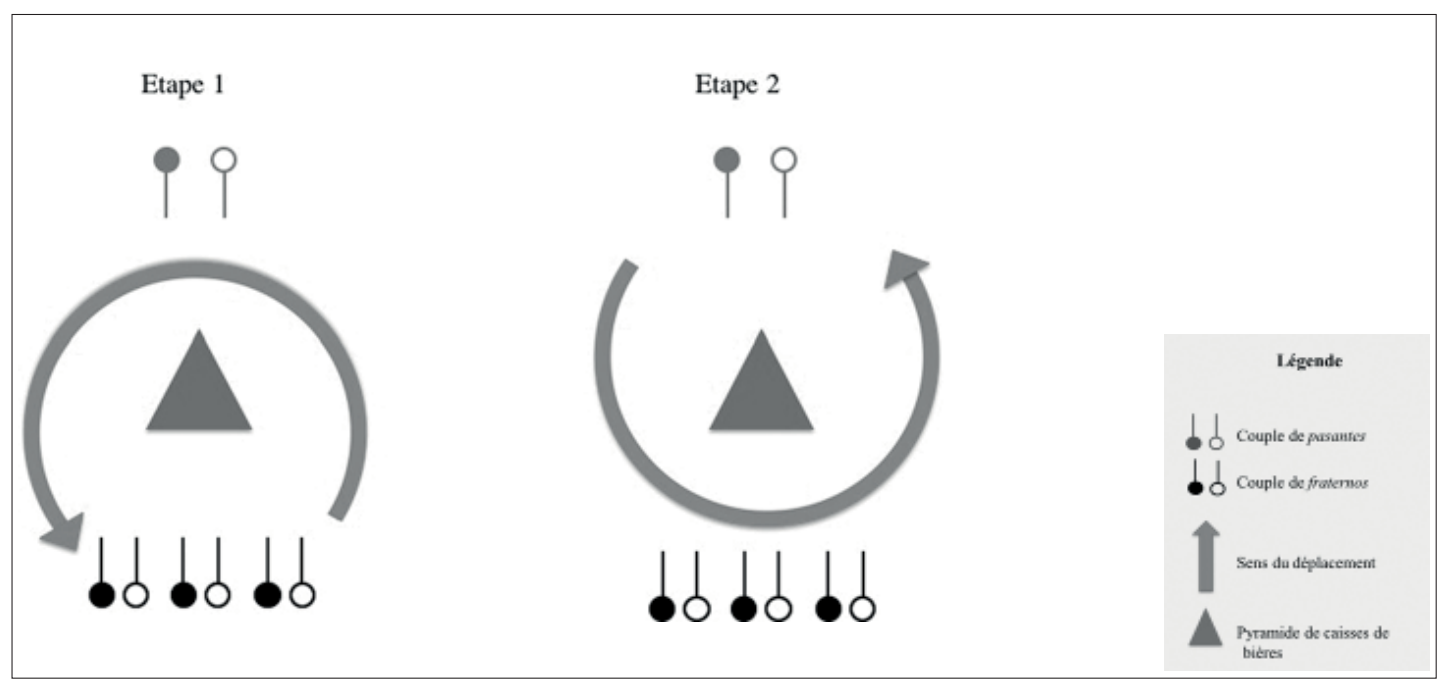

Figure 4 : Pyramide de caisses de bières et circuit de réciprocité - C Laura Fléty

Les pasantes et les fraternos se placent face à face, de part et d'autre de la pyramide. Dans un premier temps (étape 1), les fraternos passent du côté des pasantes pour leur serrer la main, les arroser de confettis et leur donner une accolade et reviennent ensuite à leur place initiale. Dans un deuxième temps (étape 2 ), ce sont les pasantes qui se déplacent du côté des fraternos et qui réalisent exactement les mêmes gestes avant de revenir à leur position initiale. Une fois ces déplacements réalisés et au signal des pasantes, tous boivent d'un trait un grand verre d'alcool, puis quittent la pyramide. Celle-ci est ensuite déconstruite et les caisses de bières sont redistribuées par les pasantes à tous les autres danseurs de la fraternidad. Plusieurs pyramides peuvent être ainsi construites successivement tout au long de la fête.

Dans ce type d'action, deux aspects sont particulièrement saillants : la symétrie et la circularité. Les fraternos comme les pasantes construisent une symétrie exacte dans leurs déplacements autour de la pyramide qui constitue le point de référence. Tout ce que font les fraternos d'un côté des caisses de bières, les pasantes le feront à nouveau de l'autre. Ici les récipients d'alcool marquent la frontière entre des groupes de statuts distincts ; les pasantes occupent la position la plus prestigieuse et les fraternos, la base du groupe de danse, sont les potentiels futurs pasantes. Si la hiérarchie est nettement verticale, les deux parties réalisent pourtant des actions marquant simultanément la notion d'équilibre et de circulation, actant ainsi une relation d'interdépendance.

Pour réussir à créer cette sensation d'équilibre, il ne suffit pas de réaliser les mêmes actions, il faut également les exécuter de la même manière, suivant un même rythme et une même énergie corporelle. Par exemple, les gestes de congratulations et de remerciements ne sont ni effusifs ni explosifs mais légèrement retenus, lents, et toujours exécutés dans une juste mesure des deux côtés. Aucun des groupes ne se distingue par une proposition gestuelle différente. Au sein de la fête, cette forme de sobriété marque l'aspect solennel de l'action en opposition avec les libations collectives, excessives et bruyantes qui se déroulent autour. Le déplacement circulaire autour des bières empilées, puis la consommation partagée et simultanée de l'alcool renvoient à la logique de l'ayni: l'un fait pour l'autre exactement ce que ce dernier a déjà fait pour lui et, à travers ce pacte, un contrat les unit. 
Cette idée de circulation est également présente lors d'autres types d'actions qui ont lieu tout au long de la fête. Elles mettront en relief le rôle des pasantes tantôt comme « destinataires », tantôt comme « donateurs » de l'alcool. Or ces rôles, encore une fois, sont matérialisés par la manière dont on met en mouvement les caisses de bières. Le rôle de donateur est incarné par une agitation corporelle et l'action d' " apporter la bière vers » alors que le rôle de destinataire se traduit par une position statique du corps et l'action de « rester sur place » pour recevoir les dons d'alcool.

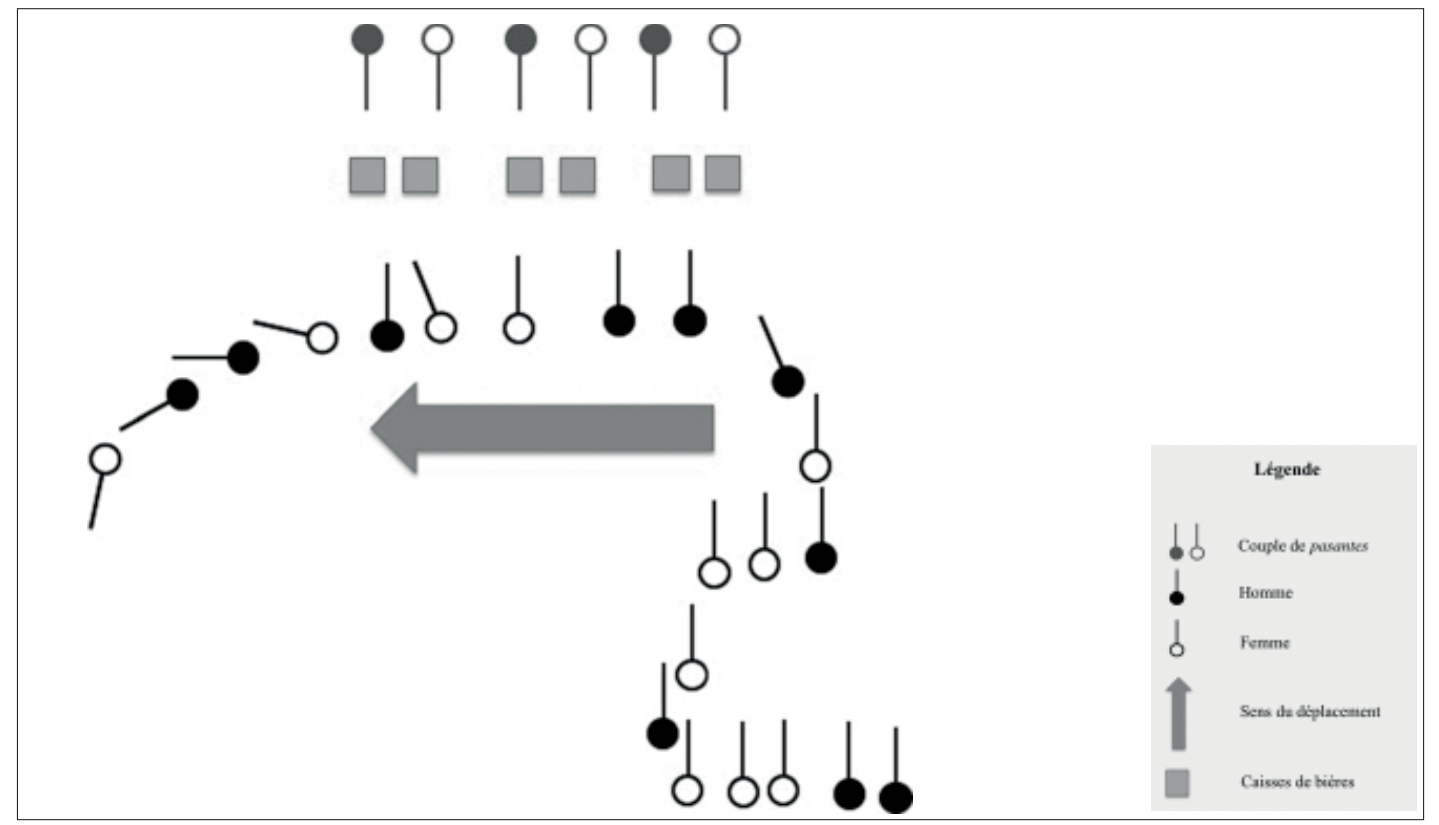

Figure 5 : Dispositif spatial avec fraternos donateurs de bières /pasantes destinataires - C Laura Fléty

Au centre de la rue, les trois couples (mari et femme) de pasantes se sont placés les uns à côté des autres. Des centaines de fraternos, hommes et femmes, affluent les uns derrière les autres pour leur serrer la main et leur offrir des caisses de bières. Ils exécutent ces actions avec empressement car la file qui s'est formée est grandissante. Par contraste avec tout ce mouvement et cette excitation collective, les couples de pasantes sont ancrés dans le sol et immobiles. Ce sont les fraternos qui passent prestement, empilant de manière énergique les caisses devant eux. Le mouvement et le don d'alcool est donc du côté des fraternos, et la fixité et l'action de recevoir, du côté des pasantes. Plus tard dans la fête, on assiste au procédé inverse (voir Figure 6). 


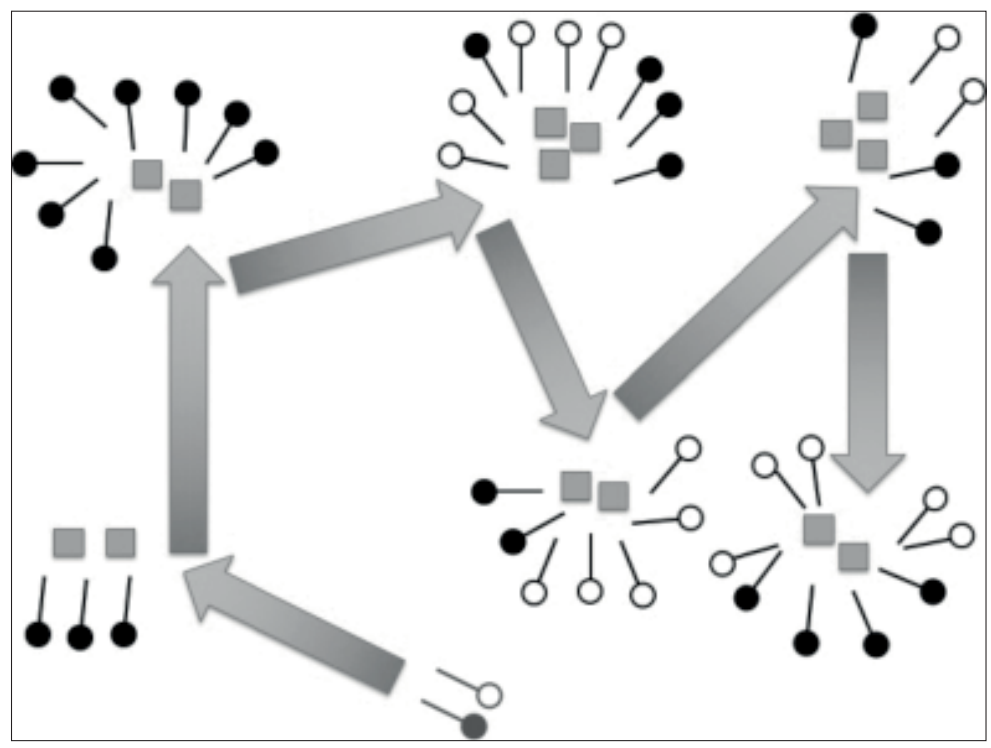

Figure 6 : Dispositif spatial avec pasantes donateurs /fraternos destinataires - (c) Laura Fléty

Cette fois, ce sont les pasantes qui vont montrer toute leur implication et « aller vers » les fraternos en leur offrant à boire. Ces derniers sont placés dans l'espace en petits groupes resserrés. Si les pasantes ont reçu précédemment les dons d'alcool dans une grande fixité (schéma précédent), ils doivent maintenant faire jouer la logique de la réciprocité avec une attitude corporelle énergique : il faut aller saluer chaque groupe de fraternos les uns après les autres pour partager des bières et placer avec emphase une multitude de caisses devant eux. Le mouvement des dons d'alcool est cette fois du côté des pasantes et la fixité/ réception du côté des fraternos.

La circulation d'alcool produit un ordre social qui rappelle les devoirs et rôles de chacun au sein de la fraternidad, tant au niveau des relations familiales et des relations de parenté élargie, qu'au niveau de la relation avec les pasantes. La réciprocité n'est pas seulement un idéal moral ou intellectuel mais est concrètement actée par les participants à travers une manière spécifique de se déplacer dans l'espace et une gestuelle qui s'organisent et rendent saillant l'objet « alcool ». D'une part, les caisses de bières deviennent les marqueurs structurants des relations sociales internes du groupe, d'autre part, elles matérialisent l'investissement économique, physique et moral de chacun dans le groupe. Par tous ces circuits de déplacement et cette activation corporelle autour des caisses, c'est la dépense d'énergie qui est valorisée, déployée à son paroxysme par la suite dans l'acte dansé. De même, par ses caisses distribuées et redistribuées qui font l'objet de constructions visuelles (pyramide, murs) montées et démontées, c'est la dépense économique collective qui est rendue visible.

L'alcool acheté en grande quantité lors de ces rassemblements qui se déroulent de manière continue sur six mois de l'année ${ }^{19}$ constitue une grande partie du budget que les danseurs consacrent à la fiesta. Cet aspect est indispensable à l'expression de la

19 Afin de préparer la célébration de Jesús del Gran Poder, les fraternos se réunissent de janvier à juin, à fréquence d'environ deux fois par mois. La nature des rassemblements exprime divers degrés de ritualité, il peut s'agir d'une répétition de danse, d'une veillée ou d'une messe catholique après lesquelles les fraternos dansent et boivent en grande quantité. 
foi religieuse car le Saint punit ceux qui « serrent leurs poches pour retenir l'argent » (Don Pedro, 49 ans, danseur de morenada) comme ceux qui « ne dansent pas pour lui » (Doña Maria, 45 ans, danseuse de morenada). On note bien ici que, dans ces deux injonctions, c'est l'immobilité de la richesse et du corps qui sont sanctionnées. L'alcoolisation collective permet la réalisation de cette double dépense indispensable tant pour réaffirmer les relations sociales internes que pour la création du lien avec le Christ Gran Poder.

\section{Où l'invitation à boire devient un piège}

Les actions et interactions qui se manifestent lors de ces rassemblements de danseurs montrent, à l'évidence, une forte continuité avec l'importance des principes de réciprocité que l'on retrouve dans le monde indigène rural, ainsi qu'une vision de l'existant dans laquelle l'effort et la dépense constituent le fondement de toute action constructive et sont à la base de la sociabilité (Cavalcanti 2007 : 6). Néanmoins si les danseurs mobilisent de manière récurrente, dans leurs discours, le concept d'ayni et la valeur positive du « partage » pour évoquer leur consommation d'alcool, rappelons que l'ayni se fonde sur l'équivalence (Dumezil 1955 ; Favre 1972 ; Molinié 1978). Or les danseurs, à travers leurs achats titanesques de caisses de bières, façonnent leur prestige social et activent d'importants mécanismes de compétition. L'alcool devient un don compétitif où l'on donne tellement que l'autre ne peut rendre, où l'on rend tellement que l'autre ne peut pas continuer le jeu (Godelier 1996). L'ayni, dans ce contexte urbain spécifique, prend donc une dimension bien différente de celle du travail communautaire des communautés indigènes qui instaure des liens égalitaires.

\section{Obliger l'autre}

Le non-respect des obligations sociales et économiques des dons de bières peut générer un tel mécontentement chez les danseurs qu'il provoque de violents conflits. Ce type d'attitude est considéré comme un affront si grand qu'il brise toute relation de réciprocité possible par la suite, et c'est alors l'ensemble de la fraternidad qui est affecté. Les dépenses d'alcool des pasantes et des fraternos répondent à un idéal de circulation de l'argent tout comme elles restent aussi une manière d'exhiber son pouvoir économique et de gagner le respect des autres danseurs.

Dans les fraternidades les plus prestigieuses, les pasantes sont ceux qui possèdent un capital économique et social conséquent : réseaux familiaux et amicaux étendus, nombreuse parenté rituelle. Jouissant d'une position confortable, ils incarnent une figure idéelle à laquelle aspirent les plus humbles danseurs. Pour afficher leur pouvoir économique et leur générosité, les pasantes mettent en scène les dons d'alcool qu'ils réservent aux fraternos. Cette exhibition ostentatoire est destinée à démontrer tout à la fois le statut et le sens du partage du pasante, la puissance économique de la fraternidad et l'offrande au monde invisible qui se concrétise par les gestes de libation pour nourrir la Pachamama et autres divinités. Les caisses de bières données marquent le statut prestigieux des pasantes et oblige les fraternos à donner quelque chose en retour. Par exemple, lorsqu'un pasante veut convaincre une famille de danser dans la fraternidad dont il a la charge rituelle annuelle, il se présentera au domicile avec plusieurs caisses de bières dont il consommera une partie avec les habitants qui ne pourront alors refuser l'invitation à danser, sous peine d'entacher l'honneur du pasante. L'honneur n'est 
pas lié à des qualités morales telles que la droiture ou l'intégrité mais témoigne d'une hiérarchie verticale. Ici, le pasante offre de la bière pour obliger à donner de la danse. Loin d'être envisagé comme un idéal égalitaire, les enjeux de l'honneur révèlent ici une volonté de puissance et impliquent une ligne de conduite selon laquelle on ne doit jamais se rabaisser devant l'autre. Cette capacité à ne pas se laisser dominer, ou « à ne jamais baisser le regard », comme le formule les danseurs, est un aspect essentiel des relations entre fraternos.

\section{Humilier par la boisson}

La scène se déroule un dimanche après-midi, sur un grand terrain de football, lors d'une répétition de danse en plein air où tous les fraternos sont présents. Deux grandes fanfares jouent et les danseurs répètent les pas de la danse morenada. Après la pratique dansée, chacun, au sein de petits groupes dispersés sur le terrain, consomme en quantité les bières prévues pour l'occasion. Une fanfare se remet alors à jouer. Plusieurs fraternos commencent à s'affairer énergiquement afin d'ériger, au centre du terrain, un grand mur formé de caisses de bières s'élevant sur plus d'un mètre. Tous les regards convergent sur cette imposante construction verticale qui devient le centre de l'attention. Les pasantes s'approchent du mur où les attend un couple marié de fraternos, visiblement très émus. Le mur constitue un don qu'ils offrent aux pasantes, avec qui ils échangent alors de longues accolades. Plusieurs autres fraternos viennent rejoindre le petit groupe et dansent autour du mur. Les donateurs vont ensuite tranquillement se rasseoir et les pasantes entament alors la déconstruction du mur, distribuant à chaque petit cercle de fraternos une ou deux caisses de bières. La scène est plutôt classique, à la différence près qu'au moment de la rencontre entre les pasantes et le couple de danseurs, la femme « donatrice » eut une attitude peu courante. Si l'émotion est habituelle dans ce genre de situation, la femme pleurait d'une façon excessive, se serrant nerveusement les mains avec une expression de profond désespoir plutôt inappropriée à la situation. J'eus quelques mois plus tard les explications de ce comportement étrange par l'un des danseurs du groupe.

Le couple de donateurs était d'anciens amis et voisins très proches des pasantes. Lorsque ces derniers assumèrent la charge de la fête, ils invitèrent le couple chez eux pour partager un grand repas, et leur offrirent plusieurs caisses de bières pour solliciter un ayni, c'est-à-dire leur participation dansée. A grand regret, le couple dut refuser car il était déjà engagé dans une autre fraternidad concurrente. Ce refus fut très mal accepté par les pasantes qui, se sentant profondément offensés, mirent le couple à la porte. Malgré cette humiliation, le couple tenta d'arranger la situation et de renouer les liens en participant à une répétition de danse, accompagné de Johny, un fraterno qui avait pour mission de jouer le rôle d'intermédiaire et de réconciliateur. Voici son récit :

Moi je connais bien ce couple, ce sont des gens très bien, je voulais les aider, je voyais bien qu'ils faisaient tête basse tu vois, ils étaient trop humiliés par toute cette situation. C'est pour ça qu'ils sont venus à la répétition avec moi, pour arranger les choses avec Eduardo, le pasante. Mais la situation a mal tournée et s'est transformée en un duel d'ego, dont chacun voulait sortir gagnant. J'étais assis avec le couple en train de boire une bière quand Eduardo est passé près de nous. Le mari a essayé de l'inviter à boire mais Eduardo a fait l'indifférent, il a esquivé, prétextant qu'il avait mille choses à faire et trop de danseurs à saluer. 
Après, moi je suis allé tout seul le rechercher pour essayer d'arranger les choses. Je lui ai dit « Eduardito viens avec nous, s'il te plait, ils veulent t'offrir une petite bière, viens t'asseoir avec nous ». Par respect, il était obligé de venir mais il a bu son verre d'un trait, sans rien dire, il n'est pas resté plus de deux minutes et il est reparti. Et là, le couple était vraiment trop offensé, tu imagines, c'était plusieurs humiliations de suite pour eux, alors ils ont voulu leur revanche. La femme était dans tous ses états, elle a déversé tous les billets qu'elle avait pliés entre ses seins, les billets sautaient sur mes genoux comme des sauterelles. Je n'arrivais même pas à compter, il y avait au moins 4000 bolivianos $^{20}$. La femme pleurait de rage et elle m'a dit de courir aller acheter cinquante caisses de bières ${ }^{21}$ et de m'occuper de monter le mur de bières, car elle voulait montrer à Eduardo qu'ils n'allaient pas se laisser humilier comme ça. Quand on a mis toutes les caisses au milieu, Eduardo et sa femme se sont approchés du mur mais je voyais Eduardo qui cherchait des yeux de qui provenait le cadeau. Quand il a vu que c'était ses voisins, on regardait tous, alors il a été obligé de les remercier. Mais ça ne s'est pas arrêté là, Eduardo ne pouvait pas les laisser reprendre le dessus comme ça. Normalement, quand on te donne cinquante caisses, tu dois en redonner la moitié ou au moins un tiers à celui qui te les a données. Mais là, Eduardo ne leur a redistribué que deux caisses pour les humilier à nouveau, plus fortement. Alors, tu sais ce qu'ont fait les voisins ? Ils n'ont pas touché ces deux caisses offertes, pas une seule goutte, ils sont restés à côté et se sont rachetés des bières à part, méprisant le cadeau humiliant d'Eduardo. Et maintenant, ils se haïssent. Ils vivent en face les uns des autres mais quand ils se croisent dans la rue, ils ne se disent plus bonjour, ni se regardent dans les yeux. (Johny)

La question du respect ne relève pas d'une dimension métaphysique mais se joue, pour les danseurs, à travers des actes aussi concrets que les dons de caisses de bières : « Humilier quelqu'un, c'est par exemple quand je t'offre une bière et que toi tu m'en donnes cinq en retour et que tu fais ça juste pour m'obliger à me préoccuper et à me dire : comment je vais à mon tour lui en rendre cinq alors que je n'ai pas d'argent? », m'explique Don Fernando, un autre danseur de morenada. Dans cet exemple, l'alcool n'est pas mobilisé pour souder une alliance, mais au contraire pour marquer distance et supériorité

Ainsi l'alcool consommé peut être un véritable signe de partage (cariño) avec sa parenté réelle et rituelle, alors qu'avec d'autres partenaires, il est à l'inverse l'enjeu d'une forte compétition où l'un devient l'obligé de l'autre et subit alors une offense. Le don d'alcool, offert lors des fêtes de la fraternidad dans le but de défier un rival, peut générer une humiliation profonde chez celui qui reçoit et ne peut pas rendre. L'achat démesuré de caisses de bières devient alors l'un des principaux moyens, pour ceux qui se sentent offensés, de se venger et de retrouver un équilibre perdu. L'alcool devient ainsi le support d'un complexe jeu de pouvoir qui détermine le prestige et la réputation de chacun.

20 Équivalent à environ 550 euros.

21 Une caisse de bières compte huit bouteilles et coûte environ 70 bolivianos, soit environ 10 euros. Cinquante caisses de bières équivalent à 3500 bolivianos, soit environ 480 euros. 


\section{Où l'alcool met les corps en mouvement}

Qu'il s'agisse de resserrer des alliances ou au contraire d'en détruire, les dons d'alcool sont des indicateurs et des créateurs efficients des relations entre fraternos. On aurait d'ailleurs tort d'opposer ici radicalement dons amical et agonistique, car tous deux contribuent, certes à des fins différentes, de la forte mobilité sociale qui caractérise le milieu des fraternidades. En effet, on y retrouve à la fois le sens du partage mais également des formes de compétitivité. Chacun doit donc apprendre, lors des fêtes, à développer une intelligence relationnelle pour créer des affinités sociales mais aussi à ne pas se laisser exclure du groupe. Or ce jeu dynamique de dons réciproques de caisses de bières qui pousse les danseurs à boire toujours plus, provoque une expérience corporelle particulière, envisagée comme indispensable à la danse et plus largement, au rituel.

\section{Boire et danser}

Si la consommation d'alcool permet une constante mise en mouvement des relations internes entre les participants, c'est aussi parce qu'en premier lieu, elle invite à la mobilité du corps. Même lorsque l'on reçoit statiquement un don de bières, ce n'est que pour le rendre par la suite de manière énergique. En outre, l'état d'ébriété ne donne pas nécessairement lieu à un laisser-aller, à un abandon de soi ou à une perte de ses moyens. Ces attitudes peuvent être des conséquences effectives de l'ivresse mais ne constituent pas un objectif en soi. Ceux qui ont des attitudes trop désinhibées lors des fêtes de la fraternidad sont d'ailleurs toujours contrôlés par le groupe qui les met à l'écart.

Lié à une situation de danse, l'alcool est surtout censé apporter une « force » (fuerza, ánimo) particulière. Cette force est conçue comme un apport énergétique qui permettrait aux participants de «tenir » la danse ${ }^{22}$. L'état d'ébriété n'exprime donc pas uniquement la recherche d'une désinhibition sociale mais relève d'une puissance particulière que le danseur dit ressentir concrètement dans son corps et qui l'amène à dépasser les limites de la fatigue. En effet, ce n'est pas sur la complexité chorégraphique - c'est à dire sur la forme et l'enchainement des mouvements - que se situe la difficulté dans la danse. L'enjeu principal ne concerne pas l'exécution virtuose d'une séquence de gestes mais une capacité psychomotrice particulière : l'endurance. Le trajet à parcourir le jour du défilé peut entraîner jusqu'à six heures de danse, quasiment en continu et dans des conditions parfois difficiles (pentes ardues, altitude, intempéries), avec des costumes opulents et inconfortables, pesant parfois plus d'une dizaine de kilos. Que ce soit la quantité d'effort physique ou la forte consommation d'alcool, il s'agit là de deux types de pratiques qui mènent à un épuisement individuel et collectif et qui éprouvent la résistance des danseurs.

22 On observe ainsi d'importantes continuités avec les fiestas des communautés indigènes dans lesquelles l'alcool est également associé à une « force » que le corps du musicien transforme en « force » musicale (Martínez 2002 : 26). Cette notion de « force » en musique étant en relation avec la capacité des sons à agir non seulement sur les êtres vivants (humains, plantes, animaux) mais également sur les entités surnaturelles (saints, entités démoniaques) (ibid. : 12). 
Personne n'oserait s'arrêter de danser par fatigue, de même qu'il serait vu très mal vu de s'arrêter de boire parce que l'on ne tient plus. Enfin, selon les danseurs, l'alcool permet d'accroître l'effort et le mouvement :

Peut-être de l'extérieur, nous donnons une mauvaise impression à ceux qui nous voient boire autant, mais ce n'est que lorsque tu danses que tu peux comprendre, on danse mieux quand on a bu, lorsque tu portes cette charge lourde sur ton dos, pendant tout le trajet, ton humeur baisse et l'alcool permet de ne pas t'affaisser, de te dépasser, l'alcool te redonne de la force pour toujours continuer à avancer (Don Felipe, 62 ans, danseur de morenada).

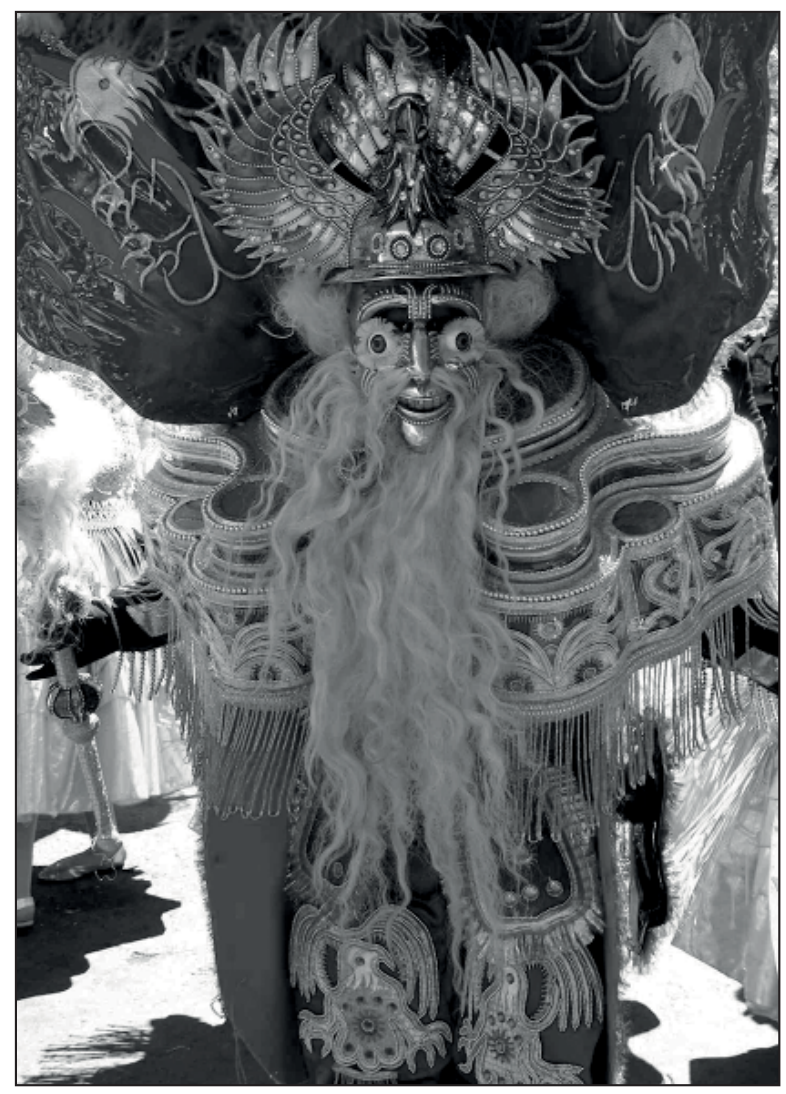

Figure 7 : Personnage de la danse morenada appelé Super Achachi (le Super Ancêtre), avec son costume ostentatoire et pesant - (C) Laura Fléty

Du point de vue de l'expérience kinesthésique, l'alcool fait danser, mais inversement la danse active aussi l'ivresse. En effet, la morenada, par ses demi-tours constants, par la perte de repères visuels qu'implique le port des masques, ou encore par l'endurance qu'elle implique, rend les corps des danseurs titubants et déséquilibrés, caractéristiques des sensations de l'ivresse. Ce rapport sensori-moteur entre danse et ivresse construit ainsi un état corporel qui est ici le moteur de l'action dévotionnelle. Le lien au sacré se matérialise dans une forme tout à fait spécifique, puisque c'est en portant une abondante matérialité, sur des corps profusément alimentés par de l'alcool, que les danseurs rendent un culte à Jesús del Gran Poder. 


\section{Conclusion}

En s'intéressant au cadre interactionnel dans lequel se déroulent les transferts de caisses de bières, j'ai montré que ces derniers matérialisaient de manière complexe plusieurs types de relations. Tout d'abord, l'alcool amorce le lien qui unit des partenaires, marque le désir de partage et d'alliance afin de densifier le réseau social de chaque danseur. A un deuxième niveau, dans la relation aux pasantes, le protocole de ces transferts impose des règles spatiales et corporelles qui renvoient aux logiques de complémentarité de l'ayni. Dans ce cas, le don d'alcool engage une contrepartie future qui instaure la relation dans la durée et préfigure de nouveaux engagements économiques et rituels envers la fraternidad, incarnés par la figure des pasantes. Enfin, à l'inverse, l'alcool peut aussi être échangé sous forme de don de compétition, annulant la réciprocité, défiant et humiliant des rivaux, ce qui ébranle alors l'unité de la fraternidad.

L'originalité de ce cas ethnographique réside sans doute dans l'entrelacement de ces différentes modalités de transferts d'alcool au cours d'un même espacetemps rituel. Cependant, quels que soient les objectifs de ces transferts de caisses de bières, il me semble qu'ils ne sont que le relais, à un deuxième plan, d'une notion beaucoup plus fondamentale : celle de la mise en mouvement continue des personnes. Par la consommation rituelle de l'alcool, c'est bien la mobilité des relations qui est constamment recherchée, entre membres d'une même famille, à une plus grande échelle entre les fraternos du groupe ou à un niveau supérieur avec les pasantes, responsables directs de la fête puisqu'ils en garantissent la réalisation et la reconduction annuelle.

En plus d'une offrande à la divinité, l'alcool, par toute l'activation corporelle qu'il mobilise, devient une force physique et relationnelle témoignant de manière particulièrement saillante de l'importance accordée chez les aymaras urbains aux principes d'échange, de dépense et d'endurance. Ces notions se matérialisent tant dans les empilements de caisses de bières, dans les circuits réalisés autour de ces constructions éphémères, dans les manières de boire et de danser. Les bouteilles de bière comme les corps enivrés des danseurs deviennent des objets activés par le rituel et participent donc, conceptuellement, à un même prototype dévotionnel. Au-delà de la désinhibition ou de la transgression qui caractérise souvent les pratiques de l'alcoolisation, notre exemple montre comment l'association tout à fait singulière entre mobilité corporelle et consommation d'alcool organise une structure sociale dont les acteurs cherchent impérativement à « mettre en actes » un principe vital de circulation.

\section{Références citées}

Abercrombie, Thomas, 1993. « Caminos de la memoria en un cosmos colonizado. Poética de la bebida y la conciencia histórica en K'ulta », in Thierry SAIGNES (éd.), Borrachera y memoria. La experiencia de lo sagrado en los Andes, pp. 139-169. La Paz : Hisbol/IFEA.

ABSI, Pascale, 2002. « Le pacte avec le diable », Journal des anthropologues, 90-91, [En ligne] <http://jda. revues.org/2213>

AlBert, Jean-Pierre, 2011. « Don, échange, argent. Quelques réflexions à partir de l'Essai sur le don de Marcel Mauss », Empan, 82, pp. 14-19.

Allen, Catherine, 1982. "Body and Soul in Quechua Thought", Journal of Latin American Lore, 8(2), pp. 179-196.

Angé, Olivia, 2011. "Yapa. Dons, échanges et complicités dans les Andes méridionales », Social Anthropology, 19(3), pp. 239-253. 
Arnold, Denise, Domingo JimÉnez et Juan de Dios Yapita, 1992. Hacia un orden andino de las cosas. La Paz : Hisbol/ILCA

Bernard, Michel, 1995. Le corps. Paris : Seuil.

Cavalcanti, Ricardo, 2007. « Las muchas naturalezas en los Andes », Periferia, 7, pp. 1-12.

Celestino, Olinda et Albert Meyers, 1981. Las cofradías en el Perú : región central. Frankfort : Vervuert.

Classen, Constance, 1990. "Sweet colors, fragrant songs: sensory models of the Andes and the Amazon", American Ethnologist, 17(4), pp. 722-735.

CSORDAS, Thomas, 1994. Embodiment and experience: the existential ground of culture and self. Cambridge: Cambridge University Press.

DumezIL, Georges, 1955. «Catégories et vocabulaire des échanges de services chez les indiens quechua : “Ayni" et "Mink'a" », Journal de la Société des Américanistes, 44, pp. 3-16.

FAVRe, Henri, 1972. Les Incas. Paris : PUF.

FLÉTY, Laura, 2015. Les cortèges de la fortune. Dynamiques sociales et corporelles chez les danseurs de morenada (La Paz, Bolivie). Thèse de doctorat en anthropologie. Université de Paris Ouest La Défense, France.

GefFroy, Céline, 2012. « La gestuelle du buveur dans le contexte festif andin (notes de recherche) », Anthropologie et Sociétés, 36(3), pp. 77-94.

Gefrroy, Céline, 2013. Boire avec les morts et la Pachamama. Une anthropologie de l'ivresse rituelle et festive dans les Andes boliviennes. Thèse d'anthropologie. Université de Nice Sophia Antipolis.

Godelier, Maurice, 1996. L'énigme du don. Paris : Fayard.

GrÉGORY, Chris A., 1982. Gift and commodities. Londres: Academic Press.

Guss, David, 2006. "The Gran Poder and the Reconquest of La Paz", Journal of Latin American Anthropology, 11(2), pp. 294-328.

Hugh-Jones, Stephen et Caroline Humphrey (eds), 1992. Barter, exchange and value. Cambridge: Cambridge University Press.

Julien, Marie-Pierre, Céline Rosselin et Jean-Pierre WARnier, 2006. Le corps : matière à décrire. Paris : Dilecta.

Marchand, Véronique, 2010. «Pollera y vestido, le langage socio-ethnique du vêtement : migration, génération, profession et instruction », Cahiers des Amériques latines, 60-61, pp. 221-239.

MARTÍnEZ, Rosalia, 2002. « Tomar para tocar, tocar para tomar. Música y alcohol en la fiesta jalq’a », in W. SANCHEz (éd.), La música en Bolivia. De la prehistoria a la actualidad, pp. 413-434. Cochabamba : Fundación Simón I. Patiño.

Martínez, Rosalia, 2009. " Musiques, mouvements, couleurs dans la performance musicale andine », Terrain, 53, pp. 84-97.

MAuss, Marcel, [1925] 1950. «Essai sur le don », Sociologie et Anthropologie, pp. 143-280. Paris : Presses Universitaires de France.

MoLINIÉ, Antoinette, 1978. « La communauté aujourd'hui », Annales, pp. 1182-1196.

RANDALL, Robert, 1993. «Los dos vasos: cosmovisión y política de la embriaguez desde el incanato hasta la colonia », in Thierry SAIGNES (éd.), Borrachera y memoria. La experiencia de lo sagrado en los Andes, pp. 73-112. La Paz : Hisbol/IFEA.

SAIGnes, Thierry, 1993. Borrachera y Memoria, la experiencia de lo Sagrado en los Andes. La Paz : HISBOL.

Stobart, Henry, 2002. "Sensational sacrifices, feasting the senses in the Bolivian Andes", in Linda Phyllis AUSTERn (ed.), Music, sensation and sensuality, pp. 97-120. New York: Routledge.

TASSI, Nico, 2010. Cuando el baile mueve montañas : religión y economía cholo-mestizas en La Paz, Bolivia. La Paz : Fundación Praia.

Testart, Alain, 2007. Critique du don. Etudes sur la circulation non marchande. Paris : Syllepse.

WaChtel Nathan, 1990. Le retour des ancêtres : Les Indiens Urus de Bolivie, XXe-XVIe siècle, Essai d'histoire régressive. Paris : Gallimard. 